\title{
SARS-CoV-2 protein encoded by ORF8 contains a histone mimic that disrupts chromatin regulation
}

\section{John Kee}

University of Pennsylvania

\section{Samuel Thudium}

University of Pennsylvania https://orcid.org/0000-0001-6670-3970

Karl Glastad

Georgia Institute of Technology

Katherine Palozola

University of Pennsylvania

Zhen Zhang

University of Pennsylvania

Yize Li

University of Pennsylvania https://orcid.org/0000-0003-1721-741X Joseph Cesare

University of Pennsylvania https://orcid.org/0000-0002-5569-1422

Rachel Truitt

University of Pennsylvania

Fabian Cardenas

University of Pennsylvania

Darrell Kotton

Boston University https://orcid.org/0000-0002-9604-8476

Konstantinos-Dionysios Alysandratos

Boston University

Wenli Yang

University of Pennsylvania

\section{Edward Morrisey}

University of Pennsylvania

\section{Benjamin Garcia}

University of Pennsylvania

\section{Susan Weiss}

University of Pennsylvania

\section{Shelley Berger}

University of Pennsylvania https://orcid.org/0000-0001-5398-4400

Erica Korb ( $\nabla$ ekorb@pennmedicine.upenn.edu ) 


\section{Biological Sciences - Article}

Keywords: SARS-CoV-2, chromatin regulation, virulence, epigenetic regulation

Posted Date: January 13th, 2021

DOl: https://doi.org/10.21203/rs.3.rs-137433/v1

License: (c) (1) This work is licensed under a Creative Commons Attribution 4.0 International License. Read Full License

Version of Record: A version of this preprint was published at Nature on October 5th, 2022. See the published version at https://doi.org/10.1038/s41586-022-05282-z. 
Title

2 SARS-CoV-2 protein encoded by ORF8 contains a histone mimic that disrupts chromatin

3 regulation

\section{Authors}

6 John Kee $\mathrm{Ka}^{\mathrm{a}, \mathrm{g}}$, Samuel Thudium ${ }^{\mathrm{a}, \mathrm{g}, \#}$, Karl Gladstad ${ }^{\mathrm{b}, \mathrm{g}, \#}$, Katherine Palozola ${ }^{\mathrm{a}, \mathrm{g}}$, Zhen Zhang $^{\mathrm{b}, \mathrm{g}}$, Yize

$7 \mathrm{Li}^{\mathrm{c}, \mathrm{ij}, \mathrm{j}}$, Joseph Cesare ${ }^{\mathrm{e}, \mathrm{g}}$, Rachel Truitt ${ }^{\mathrm{d}}$, Fabian Leonardo Cardenas ${ }^{\mathrm{d}, \mathrm{h}}$, Darrel N. Kotton ${ }^{\mathrm{k}}$,

8 Kostantinos D. Alysandratos ${ }^{k}$, Wenli Yang ${ }^{\mathrm{d}}$, Edward Morrisey ${ }^{\mathrm{d}, \mathrm{h}}$, Ben A. Garcia ${ }^{\mathrm{e}, \mathrm{g}}$, Susan R.

9 Weiss ${ }^{c, i}$, Shelley L. Berger ${ }^{b, g, f}$, Erica Korb ${ }^{a, g}$

\section{Author Affiliations}

12 Departments of Genetics ${ }^{a}$, Cell and Developmental Biology ${ }^{b}$, Microbiology $^{c}, M^{2}$ dicine ${ }^{d}$,

13 Biochemistry and Biophysics ${ }^{e}$, Biology ${ }^{\mathrm{f}}$, Epigenetics Institute ${ }^{g}$, Penn-CHOP Lung Biology

14 Institute ${ }^{\mathrm{h}}$, Penn Center for Research on Coronaviruses and Other Emerging Pathogensi',

15 Perelman School of Medicine at the University of Pennsylvania, Philadelphia, PA 19104, USA.

16 The Biodesign Institute, Center for Immunotherapy, Vaccines and Virotherapy, School of Life

17 Sciences, Arizona State University, Tempe, AZ, 85281, USA ${ }^{j}$.

18 Department of Medicine, the Pulmonary Center, Center for Regenerative Medicine Boston,

1917 University School of Medicine, Boston, MA 02118, USA ${ }^{k}$

20 Contributed equally, \#

\section{Abstract}

23 SARS-CoV-2 emerged in China at the end of 2019 and caused the global pandemic of COVID-

24 19, a disease with high morbidity and mortality. While our understanding of this novel virus is 25 rapidly increasing, gaps remain in our understanding of how SARS-CoV-2 can effectively 
26 suppress host cell antiviral responses. Recent work on other viruses has demonstrated a novel

27 mechanism through which viral proteins can mimic critical regions of human histone proteins.

28 Histone proteins are responsible for governing genome accessibility and their precise regulation

29 is critical for a cell's ability to control transcription and respond to viral threats. Here, we show that

30 the protein encoded by ORF8 (Orf8) in SARS-CoV-2 functions as a histone mimic of two critical

31 histone 3 sites containing an ARKS motif. Orf8 expression in cells disrupts multiple critical histone

32 post-translational modifications (PTMs) while Orf8 lacking this histone mimic motif does not. Orf8

33 binds to numerous histone-associated proteins and to DNA, and is itself acetylated within the

34 histone mimic site. Importantly, SARS-CoV-2 infection of multiple susceptible cell types causes

35 the same global changes of histone post-translational modifications that are disrupted by Orf8

36 expression; these include induced pluripotent stem cell-derived alveolar type 2 cells (iAT2) and

37 cardiomyocytes (iCM) and postmortem patient lung tissue. These findings demonstrate a novel

38 function for the poorly understood SARS-CoV-2 ORF8 encoded protein and a mechanism through

39 which SARS-CoV-2 disrupts host cell epigenetic regulation. Notably, this work provides a potential

40 mechanism for emerging findings from human patients indicating that ORF8 deletion results in

41 less severe illness and describes a potentially druggable pathway that may contribute to the

42 virulence of SARS-CoV-2.

\section{$44 \quad$ Main}

45 SARS-CoV-2 has proven considerably more devastating and widespread than previous virus

46 outbreaks. Recent findings indicate that several other highly virulent viruses disrupt host cell

47 epigenetic regulation through mimicry of host cell proteins ${ }^{1-3}$, particularly of histones ${ }^{4-7}$. Histones

48 function by wrapping DNA into complex structures and, in doing so, control access to the genome.

49 Histone proteins are modified by a wide-range of post-translational modifications (PTMs) that are

50 dynamically regulated to control gene expression ${ }^{8-10}$. Histone mimicry allows viruses to disrupt 
51 the host cell's ability to regulate gene expression and respond to infection effectively. Thus far,

52 only a few cases of such mimicry have been observed and validated, ${ }^{4,7}$ and no known cases of

53 histone mimicry have been found within coronaviruses. Furthermore, there are few studies

54 examining epigenetic changes associated with coronavirus infection ${ }^{11-13}$ and none yet published

55 on SARS-CoV-2. However, recent work has demonstrated that SARS-CoV-2 infection induces

56 low interferon expression indicating that it suppresses the innate antiviral host cell response ${ }^{14-16}$.

57 While SARS-CoV-2 likely employs numerous mechanisms to dampen this response, we

58 examined whether SARS-CoV-2 employs histone mimicry to disrupt histone regulation, to better

59 understand how it evades host cell antiviral responses.

60

61 To investigate whether histone mimicry is utilized by the SARS-CoV-2 virus, we first performed a

62 bioinformatic comparison of all SARS-CoV-2 viral proteins ${ }^{17}$ with all human histone proteins (Fig.

63 S1a). Most SARS-CoV-2 proteins are highly similar to those in the coronavirus strain that caused

64 the previous major SARS-CoV outbreak with the notable exception of proteins encoded by ORF3b

65 and ORF $8^{18}$. Remarkably, we detected an identical match between a region of the protein

66 encoded by ORF8 (henceforth called Orf8) and critical regions within the histone H3 amino

67 terminal tail (Fig. 1a-b, S1a-b). Furthermore, Orf8 aligns to a longer stretch of amino acids (6

68 identical, sequential amino acids) than any previously described and validated case of histone

69 mimicry $^{4,6,7,19,20}$ (Fig. S1c). Based on a crystal structure of Orf8, this region of the protein falls in

70 a disordered region that is potentially exposed to the cell in an Orf8 monomer ${ }^{21}$. Most compelling

71 is that the motif we detected contains the 'ARKS' sequence, which is found at two distinct sites in

72 the histone $\mathrm{H} 3$ tail and is well-established as one of the most critical regulatory regions within $\mathrm{H} 3$.

73 Both of these ARKS sites are modified with multiple crucial PTMs, including methylation and

74 acetylation at $\mathrm{H} 3$ Lysine 9 (H3K9me3 and $\mathrm{H} 3 \mathrm{~K} 9 \mathrm{ac})$ and at $\mathrm{H} 3$ Lysine 27 (H3K27me3 and

75 H3K27ac). Strikingly, this amino acid stretch is absent from the previous SARS-CoV virus Orf8-

76 encoded protein (both before and after a deletion generated two distinct peptides, Orf8a and 


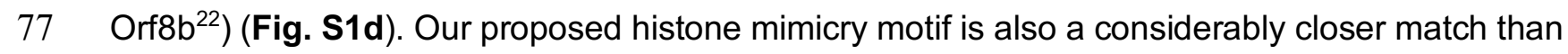

78 a previously proposed histone mimic in protein E of SARS-CoV-2 (Fig. S1e $)^{23}$. These findings

79 indicate that Orf8 may act as a histone mimic to disrupt regulation of ARKS sites on histone $\mathrm{H} 3$,

80 providing a novel mechanism through which this relatively poorly understood and highly divergent

81 protein ${ }^{24-26}$ functions during infection.

82

83 To determine whether Orf8 may act as a histone mimic, we examined whether Orf8 expression

84 disrupts histone PTM regulation using an unbiased mass spectrometry approach. HEK cells were

85 transfected with Orf8 containing a Strep tag or with a control GFP-expressing plasmid and

86 transfected cells were isolated using fluorescence-activated cell sorting (FACS). Histones were

87 purified using an acid-extraction method, and bottom-up unbiased mass spectrometry was

88 performed to quantify all detected histone PTMs. Fitting with its potential role as a histone mimic,

89 we found that numerous histone modifications were disrupted in response to Orf8 expression

90 (Table S1). We focused on significantly disrupted histone PTMs with well-defined links to gene

91 expression that contributed to at least $1 \%$ of the total peptide detected. Remarkably, we found

92 numerous histone modifications associated with active gene expression were depleted in cells

93 expressing Orf8 while histone modifications associated with chromatin compaction or

94 transcriptional repression were increased in cells expressing Orf8 (Fig. 1c). In particular,

95 modifications within the H3 ARKS motifs were highly disrupted. The peptide containing H3K9ac

96 and H3K14ac, both well-established PTMs linked to active gene expression, was decreased by

97 Orf8 expression. Conversely, the peptides containing H3K9 methyl modifications (H3K9me2 and

$98 \mathrm{H} 3 \mathrm{~K} 9 \mathrm{me} 3$ ), as well as peptides containing H3K27 methylation (H3K27me3 and H3.3K27me3,

99 both on the canonical $\mathrm{H} 3$ and variant $\mathrm{H} 3.3$ histone) were robustly increased in response to Orf8.

100 These data support a role for Orf8 as a putative histone mimic and demonstrate that it is capable

101 of disrupting histone PTM regulation at numerous critical sites within histones. 
103 Based on the pattern of histone PTM disruption observed, we hypothesized that Orf8 expression 104 decreases chromatin accessibility. We transfected HEK cells with a control construct or a plasmid 105 expressing Orf8, isolated transfected cells using FACS, and performed ATAC-sequencing 106 (ATAC-seq) to assess changes in open and closed chromatin. Orf8 expression resulted in robust, 107 global changes in chromatin accessibility, increasing chromatin compaction throughout the 108 genome including at transcription start sites (TSS) and within genic regions (Fig. 1d, S2a). To 109 define the role of the proposed Orf8 histone mimic site in chromatin regulation and to confirm 110 mass spectrometry findings through an independent approach, we again transfected HEK cells 111 with Orf8 and examined global changes in histone modifications. Here, we used 112 immunofluorescent staining with antibodies to methylated or acetylated $\mathrm{H} 3 \mathrm{~K} 9$ and $\mathrm{H} 3 \mathrm{~K} 27$ to 113 examine the modifications within the proposed histone mimic motif and that mass spectrometry 114 data indicated are disrupted by Orf8. This approach allowed for co-staining of cells with a Strep115 Tactin conjugated probe for visualization of Orf8 protein, ensuring that analyzed cells contained 116 equivalent levels of Orf8 expression for comparisons of Orf8 and Orf8-deletion constructs. We 117 found that cells expressing Orf8 exhibited increased H3K9me3 and H3K27me3 and decreased 118 H3K9ac staining compared to control plasmid transfected cells (Fig. 1e-j). To determine whether 119 these effects are due to the proposed histone mimic site within Orf8, we generated a deletion 120 construct lacking the ARKSAP histone mimic site (Orf8-del). While Orf8-del was expressed at 121 similar levels to Orf8 (Fig. S2b), it did not increase H3K9me3 or H3K27me3, and showed a trend 122 toward decreasing the effect on H3K9ac (Fig. 1e-j). Thus, the ability of Orf8 to disrupt histone 123 PTMs largely relies on the presence of the ARKSAP motif. Next, we examined another dominant 124 form of Orf8 containing an acquired mutation (S84L) commonly found in strains SARS-CoV-2. 125 This site lies outside the histone mimic region and thus is not expected to affect its ability to 126 regulate histone PTMs. We found that Orf8-S84L also increased H3K9me3 and H3K27me3, while 127 decreasing H3K9ac (Fig. S2c-e), indicating that, as predicated, this common mutation does not 128 alter the potential histone mimicry. We did not detect significant global changes in H3K27ac using 
129 these methods (Fig. S2f), potentially due to low H3K27ac basal levels and fitting with mass 130 spectrometry results.

132 To understand the mechanism through which Orf8 disrupts host cell chromatin, we began by 133 examining its intracellular localization. Notably, while Orf8 does not have a well-defined NLS, it is $13415 \mathrm{kD}$ in size and thus is small enough to diffuse into the nucleus. We first transfected HEK cells 135 with Strep-tagged Orf8 and using a cellular fractionation assay, detected Orf8 in both the 136 cytoplasm and the nucleus (Fig. 2a). We performed immunofluorescence to confirm these

137 findings through an independent approach. We found that Orf8 was present in the cytoplasm and 138 was located at the periphery of the nucleus as well as in nuclear puncta (Fig. $2 \mathbf{b}$ ). This expression 139 pattern matches those described in a previous report ${ }^{27}$, although this study focused on a 140 cytoplasmic role of Orf8. Given the observed expression pattern of Orf8, we next asked whether 141 Orf8 is associated with Lamin. We found that Orf8 colocalized with LaminB1 and LaminA/C (Fig.

142 2c, Fig. S3a). Furthermore, Orf8 bound LaminB1, histone H3, and HP1 $\alpha$, a protein associated 143 with both Lamin and histones (Fig. 2d). Similarly, reciprocal co-immunoprecipitation for LaminB1 144 and H3 confirmed Orf8 binding (Fig. 2d). These findings show nuclear localization of Orf8 and 145 indicate association with chromatin.

147 We further examined the strength of the Orf8 association with chromatin, utilizing increasing salt 148 concentrations to examine chromatin binding. We found that Orf8 dissociates from the chromatin 149 fraction at salt concentrations between those at which Lamin dissociates and the peak at which 150 histones dissociate while Orf8-del dissociates at lower salt concentrations (Fig. 2e). We next used 151 ChIP-sequencing of Orf8 itself to determine whether and where Orf8 associates with genomic 152 DNA. We discovered that Orf8 was enriched at transcription start sites and in genic regions within 153 the human genome relative to input DNA (or compared to a control ChIP performed with cells that 154 do not express Orf8) (Fig. S3b-c), although Orf8 binding does not show clearly defined peaks at 
specific genes as would be expected for an endogenous histone PTM (Fig. S3d). To confirm Orf8 association with open chromatin regions, we used ChIP-qPCR and observed greater Orf8 association with euchromatic compared heterchomatin genomic regions (Fig. S3e).

We next used targeted mass spectrometry to determine whether the proposed Orf8 histone mimic 160 site is modified similarly to histones. Using a bottom-up approach, Orf8 was purified from cells,

161 reduced, alkylated, and digested. Separation with liquid chromatography followed by parallel 162 reaction monitoring mass spectrometry (LC-PRM-MS) then targeted 8 possible unmodified and 163 modified forms of Orf8 commonly found on histones: phosphorylation on serine, then mono164 methylation, di-methylation, tri-methylation, and acetylation on lysine. Of these targets, 165 unmodified and acetylated lysine were identified. The acetylated peptide contained the $+42 \mathrm{Da}$ mass shift and demonstrated almost complete coverage of all possible product ions from the $\mathrm{N}$ terminus containing the acetyl-lysine (b ions) as well as from the C-terminus (y ions). High resolution mass spectrometry differentiated the precursor from the trimethylated peptide and matched all product ions within 10 ppm mass error (Fig. 2f, S4a). This demonstrates that Orf8 is acetylated at lysine 52 , within the proposed histone mimic site, supporting a potential function of

171 this region as a mimic of histone acetylation sites associated with active gene expression (H3K9ac

172 and $\mathrm{H} 3 \mathrm{~K} 27 \mathrm{ac})$. This finding further suggests that this motif may be recognized and modified by 173 host cell enzymes similarly to histone $\mathrm{H} 3$ and can act as a 'sink' for histone modifying enzymes.

175 Based on this observation of acetylation of OrfK52, we predicted that there may be depletion of 176 histone acetylation in proximity of Orf8. We therefore examined the localization of histone 177 modifications in cells expressing Orf8 and found that H3K9ac is depleted in regions surrounding 178 Orf8, while regions of enriched H3K9me3 typically appear at or in close proximity to Orf8 puncta 179 (Fig. S4b). This localization pattern is consistent with a role for Orf8 as a histone mimic that 180 disrupts host cell chromatin regulation causing both local and global changes in histone PTMs. 
181 Finally, we used mass spectrometry to identify additional binding partners beyond Lamin182 associated complexes (Table S2). Top hits included the HAT complex protein MORF4L, several 183 zinc finger proteins, and the transcription factor SP2 which we confirmed by co184 immunoprecipitation (Fig. S4c). Together, these results support a model in which Orf8 associates 185 with chromatin and is recognized and modified by histone acetyltransferase enzymes. It then acts 186 as a sink for host cell chromatin regulators and transcription factors, and causes both localized 187 changes in critical histone PTMs at neighboring regions within the nucleus and global disruptions 188 of chromatin regulation. These data define a role for Orf8 in disruption of host cell histone PTMs 189 through a novel case of histone mimicry of the ARKS motifs in $\mathrm{H} 3$.

191 Next, we examined Orf8 and histone PTM regulation in the physiological context of viral infection 192 and in a cell type relevant to the COVID-19 disease state using the A549 lung derived cell line 193 expressing the ACE receptor (A549 $\left.{ }^{A C E}\right)$. We obtained a highly specific antiserum for Orf8 (Fig. 194 S5a-b) and stained A549ACE cells infected with SARS-CoV-2 for Orf8 and with J2 antibody for 195 dsRNA, an intermediate in the replication of SARS-CoV-2 RNA, to identify infected cells. We 196 detected Orf8 in both the cytoplasm and nuclear periphery of infected cells where it colocalized 197 with LaminA/C (Fig. 3a, S5b), similar to the patterns observed in cells transfected with Orf8 (Fig. 198 2a-c). The requirement for viral inactivation through methods such as fixation prevented 199 subsequent biochemical analysis of virally expressed Orf8. However, we confirmed that in 200 A549ACE cells exogenously expressing Orf8, sequential salt extractions showed similar Orf8 201 chromatin association as in HEK cells (Fig S5c) and similar Orf8 localization (Fig. S5d) as in HEK 202 cells. To determine whether similar chromatin disruptions occur in the context of viral infection as 203 we detected in response to Orf8 expression, we infected A549ACE cells with SARS-CoV-2 or 204 performed a mock infection with an MOI of 1 and fixed and stained cells for H3K9 and H3K27 205 modifications 48 hours after infection. SARS-CoV-2 infection increased H3K9me3 and 206 H3K27me3 and decreased H3K9ac (Fig. 3b-g), replicating the effects of Orf8 expression. 
207 Together, these data indicate that both Orf8 expression and SARS-CoV-2 infection result in global 208 changes in histone regulation and chromatin accessibility, providing a novel mechanism through 209 which SARS-CoV-2 can disrupt host cell function.

211 New data from COVID-19 human patients, recently published in The Lancet, found that a 382212 nucleotide deletion variation in SARS-CoV-2 that blocks expression of the ORF8 gene (Fig. 4a) 213 is associated with a milder infection in COVID-19 patients ${ }^{28}$. Furthermore, Orf8 expression has

214 been shown to block type 1 interferon and NF-kB responsive promoters and to inhibit induction of 215 interferon-stimulated genes during viral infection ${ }^{29}$. To determine whether disruption of chromatin 216 and transcriptional regulation could contribute to the lack of a robust interferon response, we

217 performed RNA-sequencing to determine how gene expression is disrupted by SARS-CoV-2 218 infection in A549 ${ }^{\mathrm{ACE}}$ cells. We found that, despite widespread differential gene expression (Fig.

219 S6a-b), interferon viral response genes were only mildly induced by infection as measured by 220 gene ontology analysis, overlap with a defined set of A549 interferon response genes ${ }^{30}$, or

221 examination of specific response genes (Fig. S6b-e). These data support recent findings 222 indicating SARS-CoV-2 results in an imbalanced host response with a limited interferon 223 response ${ }^{14-16}$.

225 To examine site-specific histone PTM regulation at interferon response genes and to determine 226 whether the global chromatin disruptions that we observed in A549 cell lines occur in cell types 227 that are highly relevant to COVID-19 disease, we analyzed chromatin regulation of lung host cells 228 by infecting human induced pluripotent stem cell-derived lung alveolar type 2 (iAT2) pulmonary 229 cells. This is a highly disease-relevant cell type similar to the lung cells infected in human patients 230 that express all of the major markers of primary human AT2 cells ${ }^{31}$. We then used ChIP231 sequencing with ChIP-RX normalization (Fig. S7a) to allow for detection for global changes in 232 histone PTMs. Strikingly, we found that infected iAT2s showed globally increased H3K9me3 and 
233 H3K27me3 and decreased H3K9ac (Fig. 4b), again matching the effects of Orf8 expression. In 234 addition to global changes, increased $\mathrm{H} 3 \mathrm{~K} 9 \mathrm{me} 3$ and $\mathrm{H} 3 \mathrm{~K} 27 \mathrm{me} 3$ and decreased H3K9ac were 235 found at interferon-stimulated genes such as IFITM2, ADAR and FOSL2 (Fig. 4c), indicating a 236 potential chromatin-based mechanism that dampens induction of key response genes during viral

237 infection. In addition, we carried out ChIP-sequencing experiments in human induced pluripotent 238 stem cell-derived cardiomyocytes (iCMs) to determine if similar effects are observed in a second 239 cell type that is sensitive to SARS-CoV-2 infection. We again observed the same global changes 240 in histone PTMs found in response to Orf8 expression and in response to infection in A549ACE 241 cells and iAT2 cells (Fig. S7b).

243 Lastly, we obtained postmortem lung tissue samples from three COVID-19 patients and matched 244 controls. We stained tissue for SARS-CoV-2 Nucleocapsid protein to identify infected cells and 245 for H3K9me3 to measure histone PTM changes. We found that in all patient samples, infected 246 cells showed increased $\mathrm{H} 3 \mathrm{~K} 9$ me3 staining compared to neighboring cells within the same tissue

247 as well as compared to control tissue (Fig. 4d-e, S7c). While limited sample availability limits the 248 conclusions that can be drawn from this assay, this finding indicates that histone PTMs are also 249 disrupted in patients with severe COVID-19 disease. Furthermore, this result supports data 250 obtained from exogenous Orf8 expression and infection of A549 ${ }^{\mathrm{ACE}}$, iAT2, and iCM cells showing 251 dramatic changes in histone PTM regulation. Together, our data examining the specific role of 252 the Orf8 protein in disrupting chromatin regulation and the equivalent widespread chromatin 253 disruptions in response to SARS-CoV-2 infection, provide a mechanism that explains differential 254 disease progression and severity in patients with a deletion of the Orf8 coding region in the SARS255 CoV-2 genome.

257 The work described here identifies a novel case of histone mimicry in the SARS-CoV-2 virus and 258 defines a mechanism through which SARS-CoV-2 acts to disrupt host cell chromatin regulation. 
We found that the protein encoded by the SARS-CoV-2 ORF8 gene contains an ARKS motif and

260 that Orf8 expression disrupts histone PTM regulation. Orf8 is associated with chromatin-

261 associated proteins, histones, DNA and the nuclear lamina both in the context of exogenous

262 expression and virally infected cells. Furthermore, Orf8 is itself acetylated within the histone mimic

263 motif similarly to histones. SARS-CoV-2 infection results in identical global histone PTM

264 disruptions as Orf8 expression in numerous cell types including A549ACE cells, iAT2s, iCMs, and

265 COVID-19 human patient lung tissue. Finally, this work provides a molecular basis for the recent

266 discovery that patients infected with a form of SARS-CoV-2 containing a deletion in the gene

267 encoding Orf8 have less severe illness and better outcomes ${ }^{28}$.

269 Ultimately, these findings explain the function of the previously poorly understood and highly 270 divergent SARS-CoV-2 protein Orf8. In addition, given that many epigenetic pathways and 271 histone modifying enzymes are druggable, in many cases with therapeutics already approved for 272 use in humans, this work suggests potential avenues for the development of treatments that target 273 epigenetic pathways. Finally, these data have critical implications for our understanding of 274 COVID-19 pathogenesis in patients and emerging viral strains carrying deletions and mutations 275 in the ORF8 gene.

\section{Author contributions}

278 J. Kee designed, performed, and analyzed the majority of the experiments. S. Thudium generated 279 cells, samples, and DNA constructs. K. Palozola performed ATAC-seq and generated samples 280 for histone PTM analysis. K. Gladstad and Z. Zhang performed and analyzed ChIP-sequencing 281 experiments. J. Cesare performed and analyzed mass spectrometry experiments with guidance 282 from B.A. Garcia. R. Truitt generated iCM cells with guidance from W. Yang. F.L. Cardenas, 283 generated iAT2 cells with guidance from E. Morrisey. D.N. Kotton and K.D. Alysandratos provided 284 stem cell lines. Y. Li performed SARS-CoV-2 viral infections. S.R. Weiss provided input and 
285 expertise and lead viral work. S.L. Berger provided input and expertise and lead ChIP-sequencing

286 studies. E. Korb lead the project and wrote the manuscript.

287

288 Acknowledgements

289 We thank and acknowledge M Weitzman for feedback, A. Stout for microscopy support, R. Jain

290 and P. Shaw for protocols and suggestions, and M. Feldman and K. Montone for providing

291 patient samples.

292 
a

\begin{tabular}{|c|c|c|c|c|c|c|c|c|}
\hline & \\
\hline & Control 1 & Control 2 & Control 3 & Control 4 & Orf81 & Orf8 2 & Orf83 & Orf8 4 \\
\hline \multirow{2}{*}{$\begin{array}{c}\text { H3K18acK23ac } \\
\text { H3K4me1 }\end{array}$} & 0.80068 & 1.880615 & 0.234161 & 0.644073 & -0.559978 & -0.940955 & -1.16094 & -0.897656 \\
\hline & 0.576166 & 1.297697 & 1.287512 & -0.001907 & -0.679918 & 0.076346 & -0.733937 & -1.821961 \\
\hline H3K9acK14ac & 0.786249 & 1.318883 & 0.977602 & 0.721088 & -1.316418 & -0.860969 & -1.243227 & -0.383209 \\
\hline Н3К23ас & 0.659069 & 1.690396 & 0.644259 & 0.635589 & -1.012039 & -0.316323 & -1.393043 & -0.907907 \\
\hline H4K20me1 & 0.771179 & 0.921831 & 1.387971 & 0.583132 & -1.206511 & -0.08108 & -1.150621 & -1.225901 \\
\hline H2AK5ac & 0.041774 & 0.861665 & -0.047662 & 2.085614 & -1.154831 & -0.337014 & -1.175288 & -0.274258 \\
\hline H3K36me3 & 1.259605 & 0.627705 & 1.512703 & 0.151656 & -0.980022 & -0.972435 & -1.311124 & -0.288089 \\
\hline \multirow{4}{*}{$\begin{array}{cc}\text {. } & \begin{array}{c}\mathrm{H} 3.3 \mathrm{~K} 27 \mathrm{me} 3 \\
\mathrm{H} 3 \mathrm{~K} 27 \mathrm{me} 3\end{array} \\
\mathrm{H} & \mathrm{KK} 9 \mathrm{me} 3 \\
\mathrm{H} 3 \mathrm{~K} 9 \mathrm{me} 2\end{array}$} & -0.692592 & -0.486838 & -1.898896 & -0.098886 & 0.141373 & 0.398789 & 1.242089 & 1.394961 \\
\hline & -0.778962 & -1.481705 & -0.106208 & -0.913679 & 1.280317 & 0.018172 & 1.602525 & 0.379539 \\
\hline & -0.605585 & -1.687834 & -0.491815 & -0.710808 & 0.739529 & 0.12616 & 1.228119 & 1.402233 \\
\hline & -0.972711 & -1.305275 & -0.549947 & -0.623706 & 0.40938 & 0.09307 & 1.732982 & 1.216207 \\
\hline
\end{tabular}

b Orf 8 ...RVGARKSAPLIE...

H3. 1 .. TKAARKSAPSTG ...

H3.2 ... TKAARKSAPATG ...

H3. 3 ... TKAARKSAPATG . . .

H3К'27

Fold Change

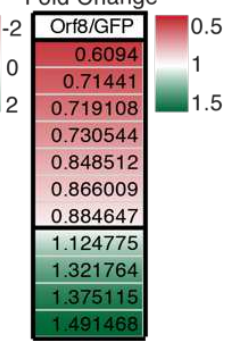

H3. 2 ... KQTARKSTGG...

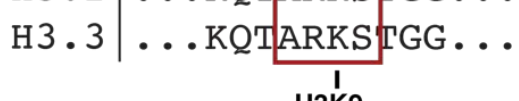

d

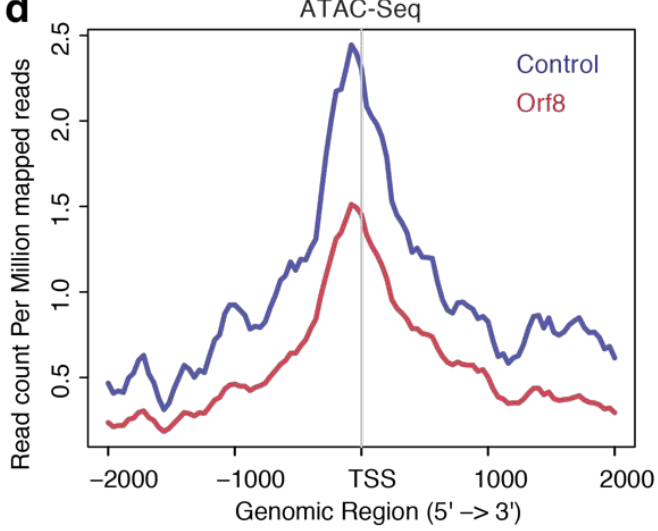

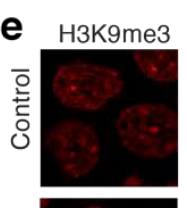

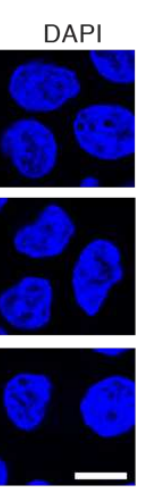

f H3K9me3
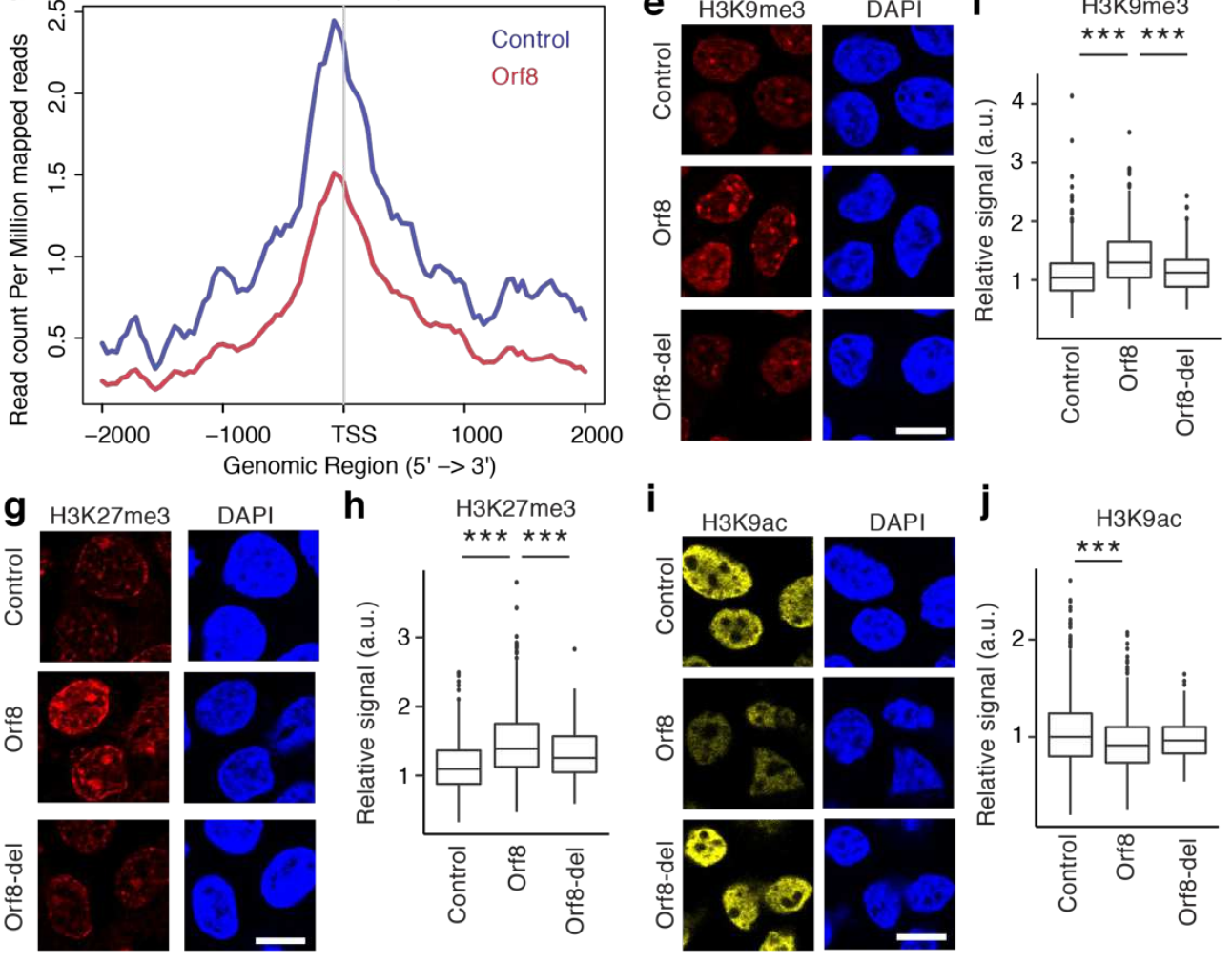

Figure 1. Orf8 histone mimicry and localization. (a,b) Orf8 contains an ARKS motif that

296 matches the histone $\mathrm{H} 3$ tail regions surrounding the critical sites H3K9 (a) and H3K27 (b). (c)

297 Mass spectrometry analysis of histone PTMs in control (GFP) or Orf8 expressing HEK cells

298 isolated by FACS. Z-score and fold changes are shown for modifications that are significantly

299 changed in response to Of8 expression, were detected over a minimal threshold of $1 \%$ of the total 
300 peptide abundance, and have well-established functions. Full results and raw data are shown in 301 Table S1. (d) ATAC-sequencing of 2 independent replicates of HEK cells expressing GFP or Orf8 302 isolated by FACS. Reads per million mapped surrounding the transcription start site (TSS) of all 303 genes are averaged. (e-j) HEK cells transfected with GFP or Strep-Orf8 show that Orf8 304 expression increases H3K9me3 (e-f) and H3K27me3 (g-h) while decreasing H3K9ac (i-j). 305 Conversely, Orf8 with a deletion of the histone mimic site ARKSAP (Orf8-del) does not affect 306 these histone PTMs. N = 614 (GFP), 497 (Orf8), 170 (Orf8-del) cells for H3K9me3; 616, 550, 154 307 cells for H3K27me3; 666, 568, 170 cells for H3K9ac compiled from 3 independent transfections. $308^{* * *}, p<0.001,1$-way ANOVA with post-hoc 2-sided t-test and Bonferroni correction. Scale bars = $3095 \mu \mathrm{M}$. 


\section{Figure 2}
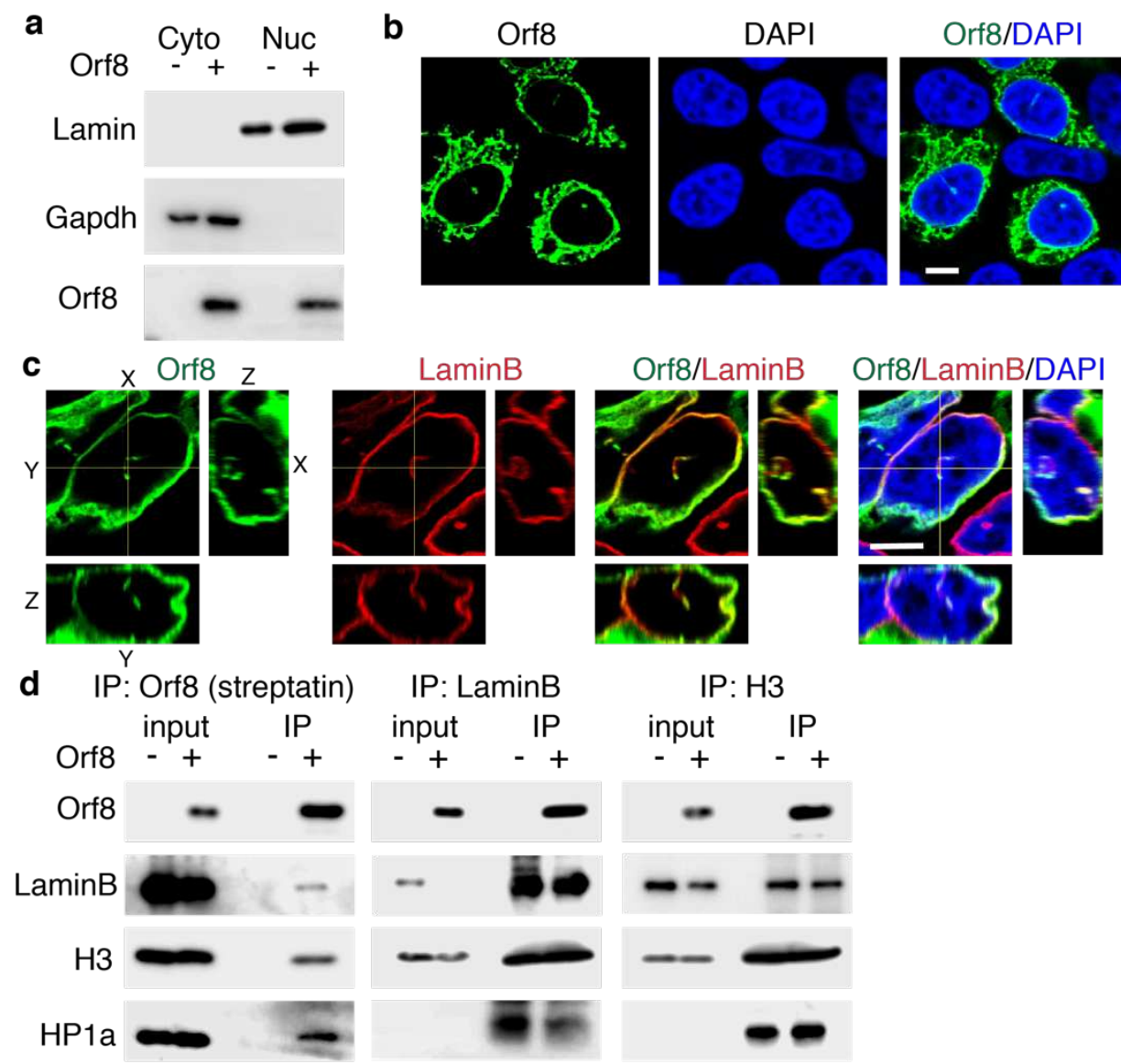

e
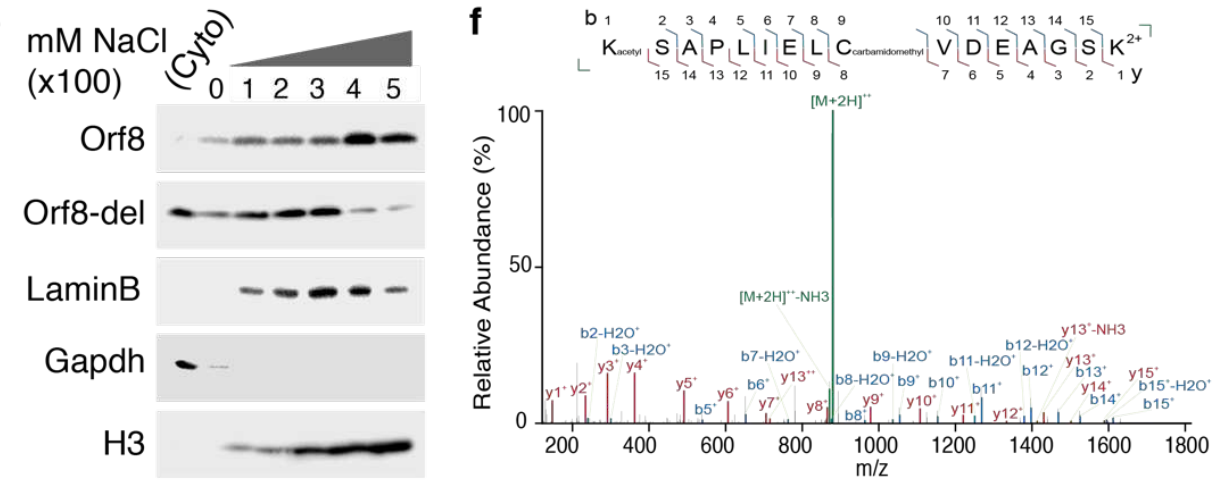

313 Figure 2. Mechanism of Orf8 function. (a). Subcellular fractionation of HEK cells transfected

314 with Strep-Orf8 indicates Orf8 is present in the cytoplasm and nucleus. (b) Staining of HEK cells

315 transfected with Strep-Orf8 shows Orf8 is expressed in the cytoplasm and at the nuclear periphery

316 as well as in nuclear puncta. (c) Orf8 colocalizes with Lamin at the nuclear periphery and within

317 nuclear puncta. Images shown include rotation of z-stacks (right and bottom panel for each stain) 
318 to demonstrate colocalization throughout the nucleus. (d) Orf8 co-immunoprecipitates with Lamin

319 complex-associated proteins including LaminB, HP1, and H3. Streptactin-conjugated beads were

320 used for Orf8 IPs. '- indicates cells that are not expressing Orf8 for negative control IPs performed

321 in parallel. (e) Sequential salt extraction of HEK cells expressing Orf8 indicates Orf8 is present in

322 chromatin fractions and dissociates from chromatin between peaks of dissociation for LaminB

323 and histone protein H3. 'Cyto' indicates cytoplasmic fraction. (f) Targeted mass spectrometry

324 analysis of trypsin-digested Orf8 shows Orf8 is acetylated at lysine 52 , at the site of the proposed

325 histone mimic in Orf8. The intact $2+$ charged peptide or precursor at $879.9508++\mathrm{m} / \mathrm{z}$ was isolated

326 and fragmented resulting in the MS/MS spectra shown. After fragmentation, the MS/MS spectra

327 show unfragmented precursor (green) with matching product ions (b ions in blue, y ions in red)

328 within 10ppm mass error. Each fragment's intensity is given relative to the highest ion in the

329 MS/MS spectra across the $\mathrm{m} / \mathrm{z}$ range. The color, letter, and number of each fragment indicates

330 the sequence that fragment contains within the larger peptide (top). $Y$ fragments (red) indicate C-

331 terminus matched fragments. B fragments (blue) indicate $\mathrm{N}$ terminus matched fragments. Scale

332 bars $=5 \mu \mathrm{M}$. 
Figure 3
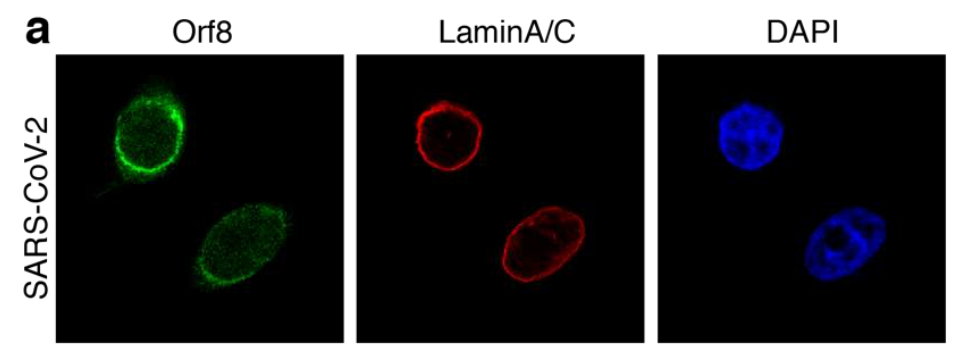

Orf8/LaminA/C
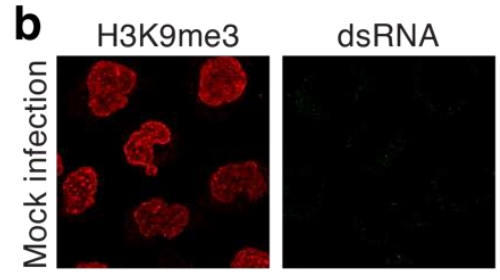

\section{DAPI}

H3K9me3/dsRNA
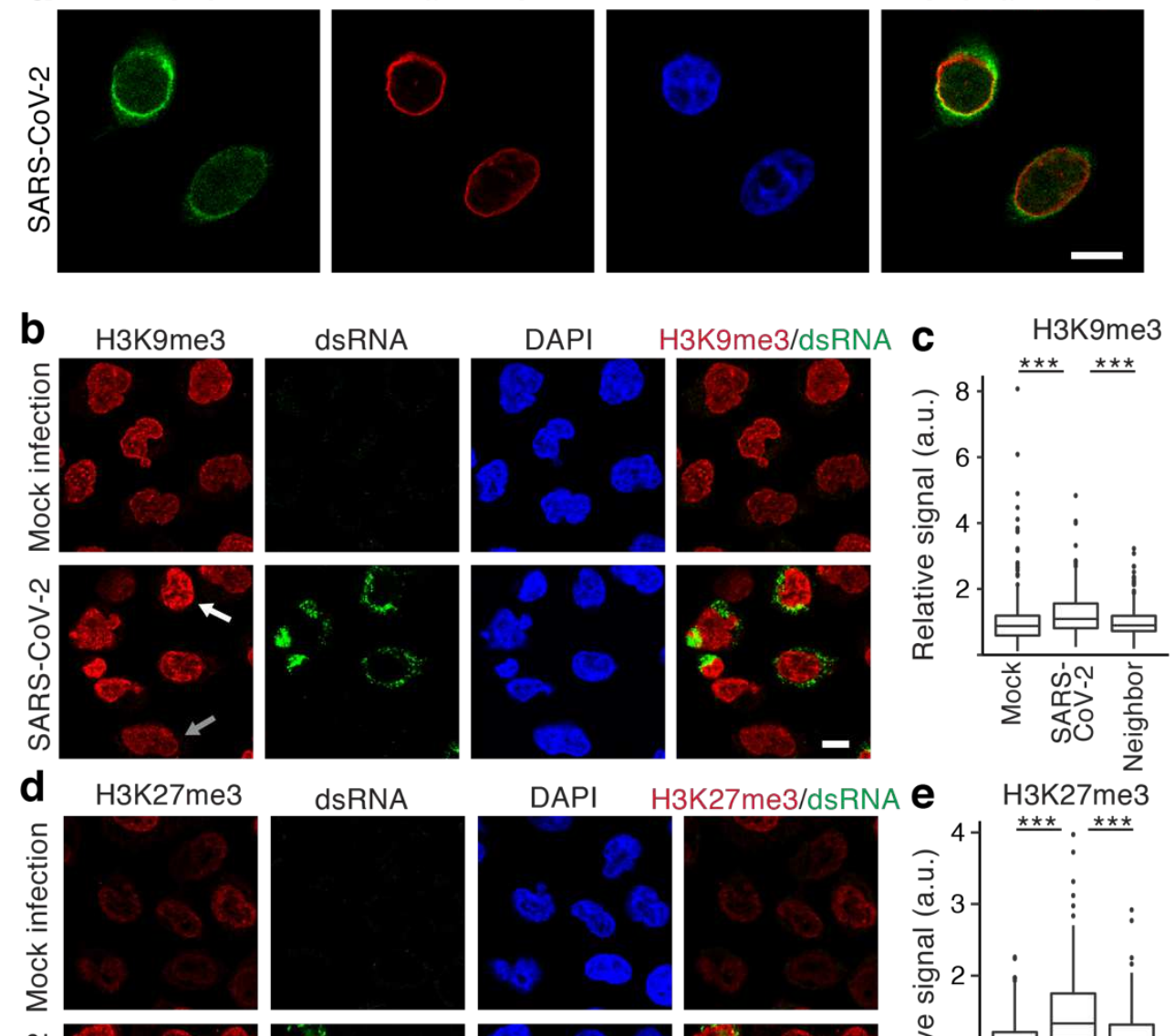

dsRNA

DAPI H3K27me3/dsRNA $\boldsymbol{e}$
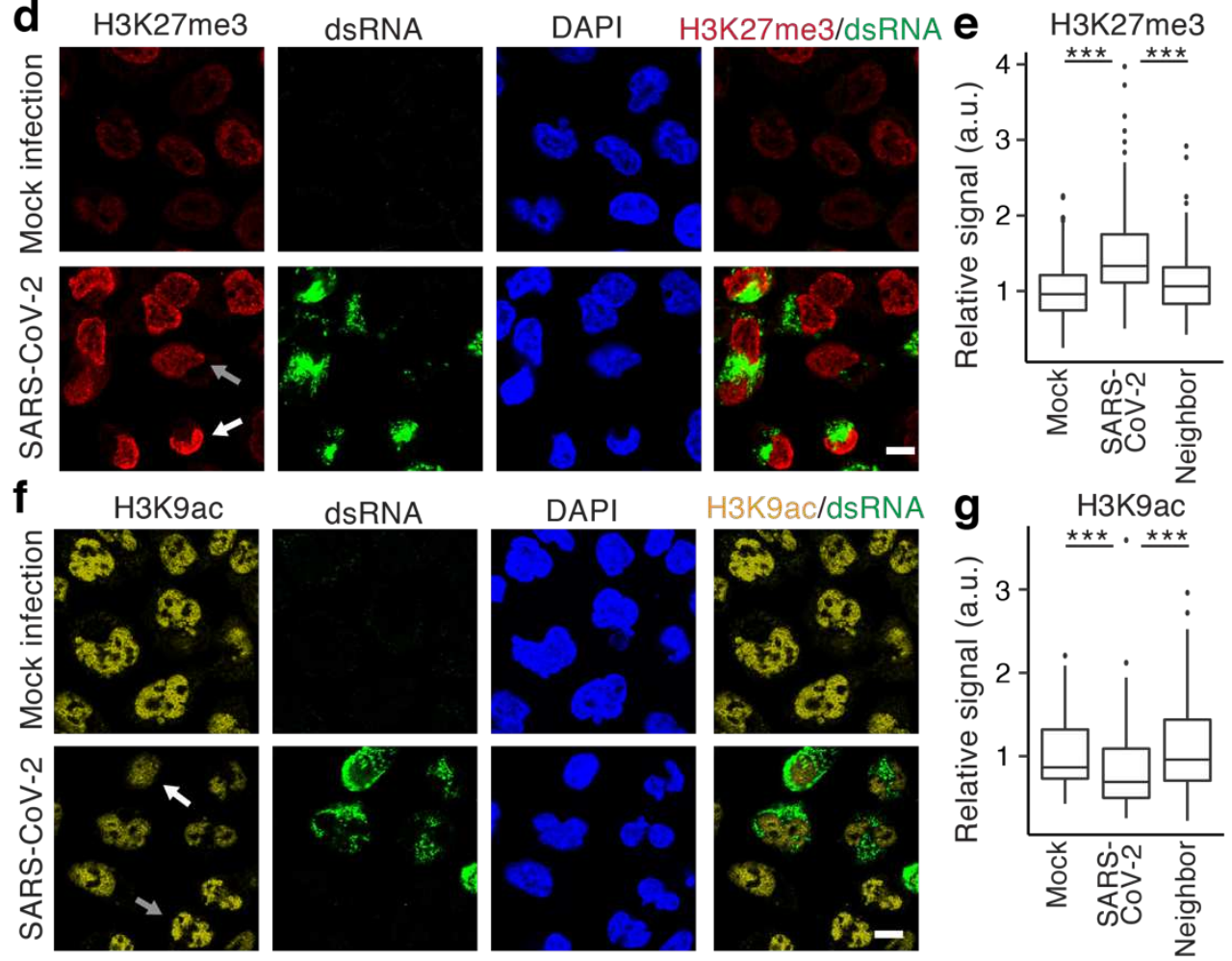

H3K9ac/dsRNA
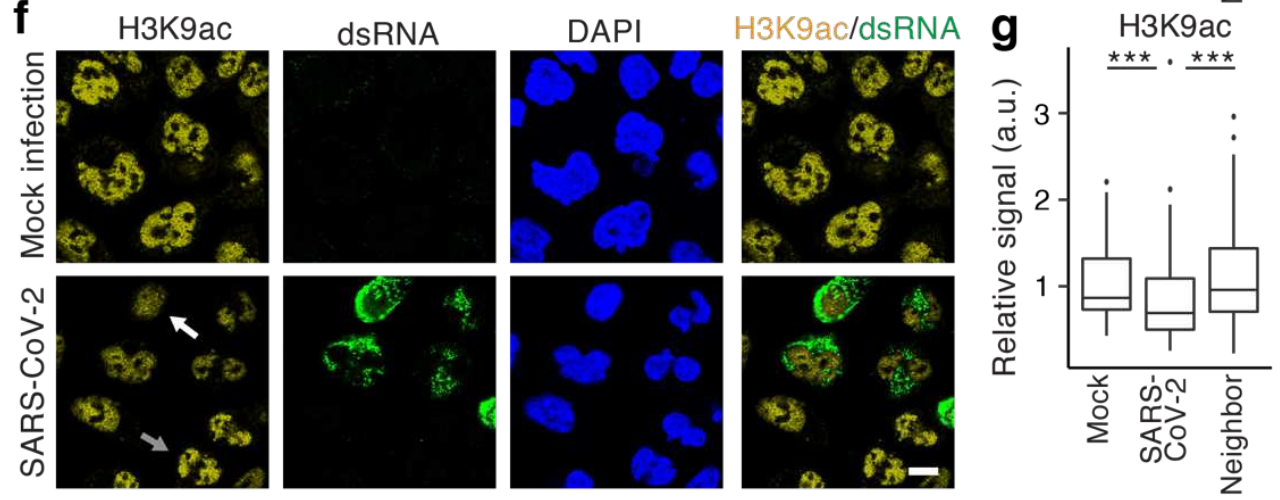

336 Figure 3. SARS-CoV-2 infection affects histone PTMs. (a) Orf8 and LaminA/C staining of

337 SARS-CoV-2 infected ACE ${ }^{\mathrm{A} 549}$ cells at $\mathrm{MOI}=1,48$ hours post infection. (b) $\mathrm{H} 3 \mathrm{~K} 9 \mathrm{me} 3$ staining of 
338 ACE $^{\text {A549 }}$ cells 48 hours after SARS-CoV-2 or mock infection at MOI=1. (c) Quantification of 339 H3K9me3. $\mathrm{N}=475$ (Mock), 158 (SARS-CoV-2), 265 (Uninfected neighbor) cells per condition 340 from 3 independent infections. (d) H3K27me3 staining of ACE ${ }^{\text {A549 }}$ cells 48 hours after SARS-CoV3412 or mock infection at MOI=1. (e) Quantification of H3K27me3. N= 455 (Mock), 133 (SARS-CoV342 2), 250 (Uninfected neighbor) cells per condition from 3 independent infections. (f) H3K9ac 343 staining of $\mathrm{ACE}^{\mathrm{A} 549}$ cells 48 hours after SARS-CoV-2 or mock infection at $\mathrm{MOI}=1$. (g) 344 Quantification of H3K9ac. N= 385 (Mock), 177 (SARS-CoV-2), 268 (Uninfected neighbor) cells 345 per condition from 3 independent infections. White arrows indicate infected cells. Grey arrows 346 indicate uninfected neighbors. ${ }^{* *}, \mathrm{p}<0.001,1$-way ANOVA with post-hoc 2-sided t-test and 347 Bonferroni correction. Scale bars $=5 \mu \mathrm{M}$. 

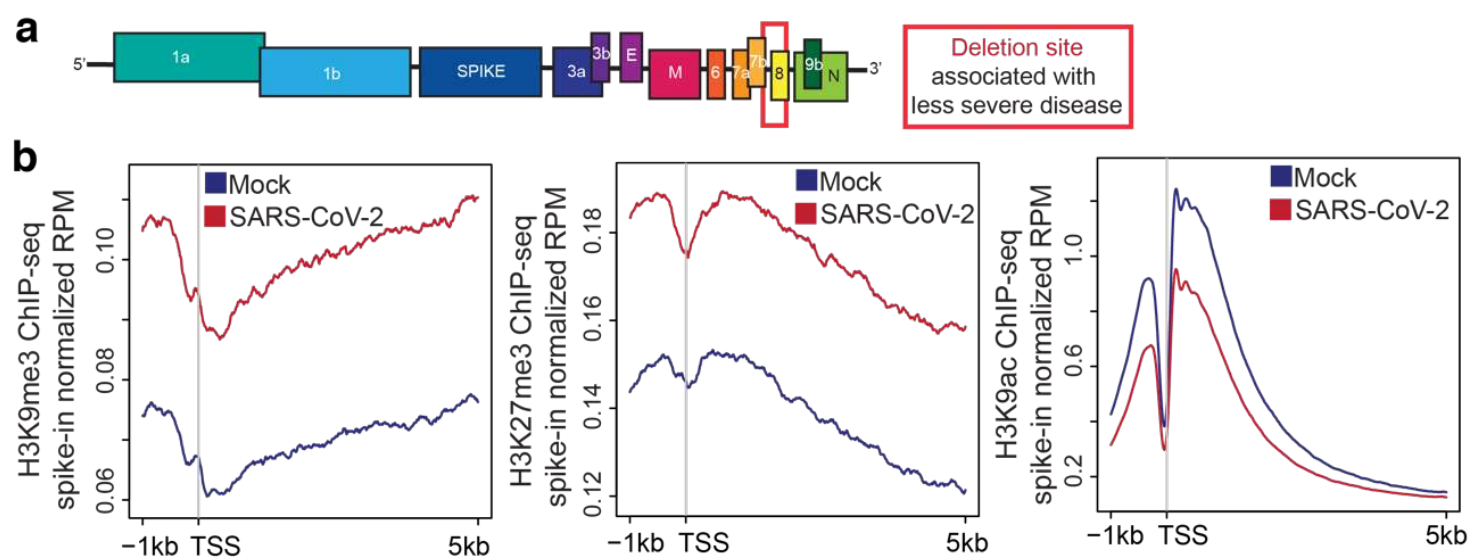

\section{C}
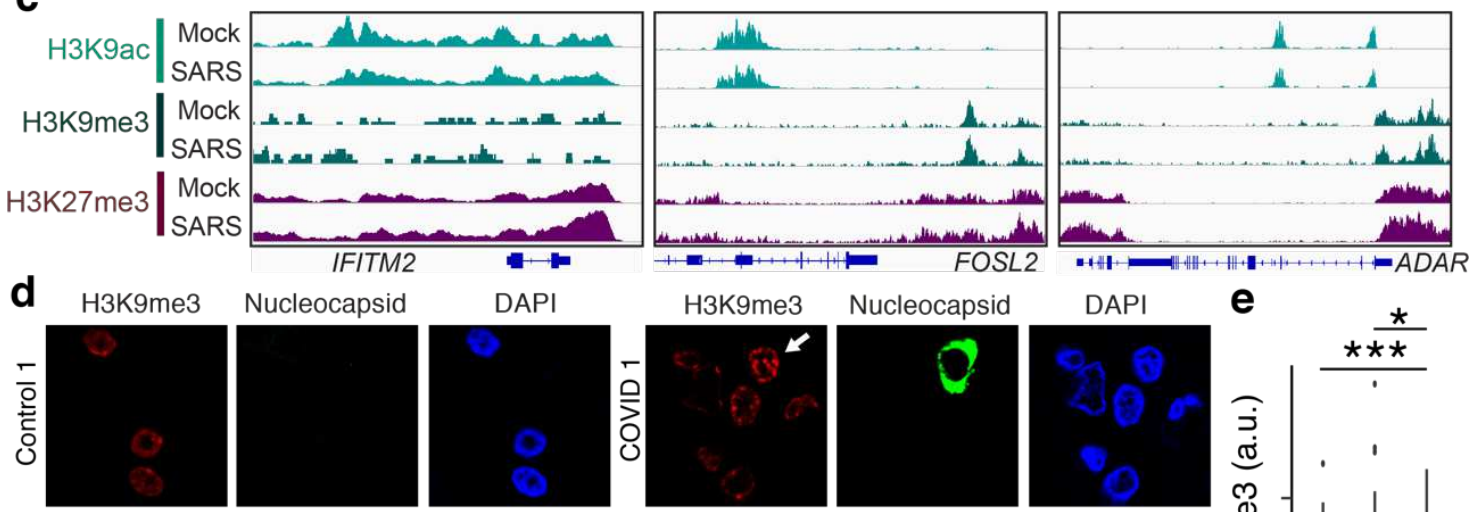

DAPI

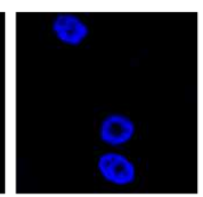

H3K9me3
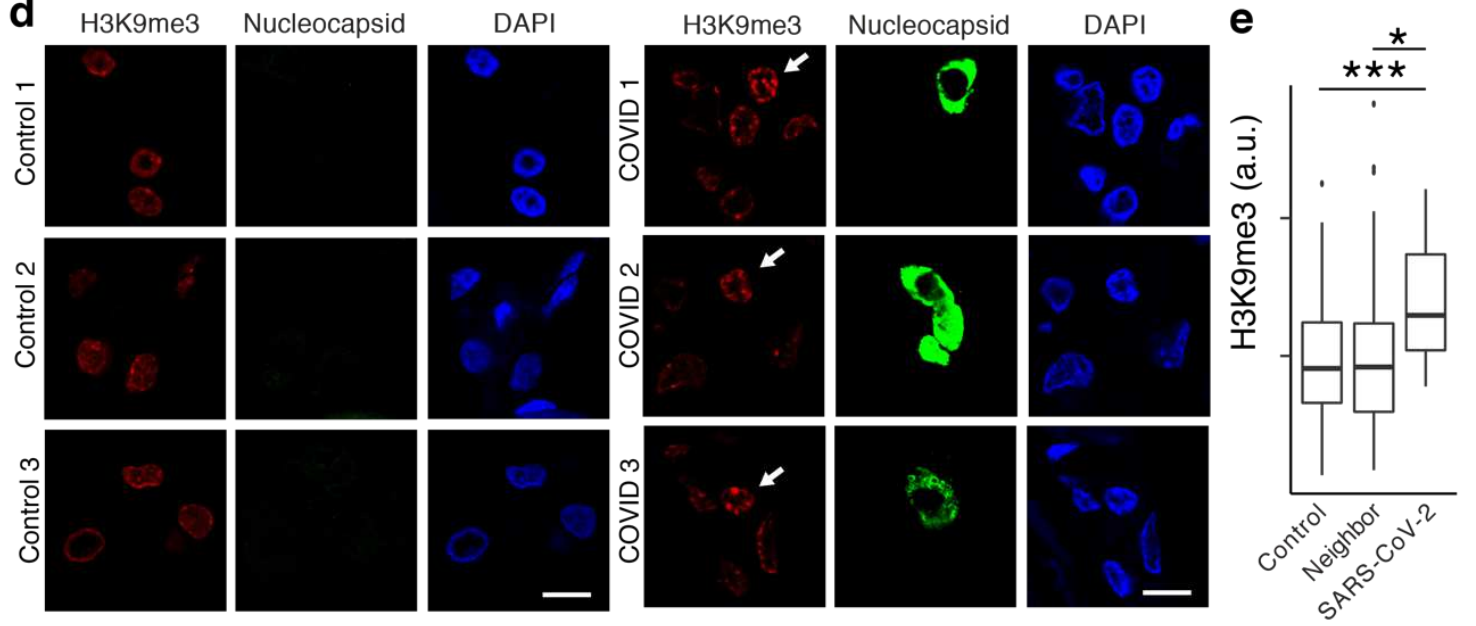

351 Figure 4. Genome-wide sequencing demonstrates global changes in histone PTMs in

352 response to SARS-CoV-2 infection. (a) Model of the SARS-CoV-2 genome indicating the site

353 of deletion that decreases disease severity in COVID-19 patients. (b) ChIP-sequencing of iPSC

354 derived iAT2 cells fixed by 4\% PFA 48 hours after SARS-CoV-2 or mock infection at MOI=5.

355 ChIP-RX normalization shows globally increased H3K9me3 and H3K27me3 and decreased

356 H3K9ac. $\mathrm{N}=$ at least 3 infections per ChIP fixed by 4\% PFA 48 hours after SARS-CoV-2 or

357 mock infection at $\mathrm{MOI}=5$. (c) ChIP-sequencing gene tracks for interferon response genes with 
358 limited responses to SARS-CoV-2 infection. (d) Postmortem COVID-19 patient lung tissue

359 stained for H3K9me3 and Nucleocapsid protein to identify SARS-CoV-2 infected cells. (e)

360 Quantification of $\mathrm{H} 3 \mathrm{~K} 9 \mathrm{me} 3$ in infected cells compared to neighboring cells from the same tissue

361 slice. $\mathrm{N}=12$ SARS-CoV-2 infected cells and 131 uninfected neighboring cells from 3 COVID

362 patient samples and 60 cells from 3 control patients. 1-way ANOVA with post-hoc 2-sided t-test

363 and Bonferroni correction. Scale bar $=10 \mu \mathrm{M}$.

364 


\section{References:}

445 1. Elde, N. C. \& Malik, H. S. The evolutionary conundrum of pathogen mimicry. Nat. Rev.

$446 \quad$ Microbiol. 7, 787-797 (2009).

447 2. Davey, N. E., Travé, G. \& Gibson, T. J. How viruses hijack cell regulation. Trends Biochem. 448 Sci. 36, 159-169(2011).

449 3. Chaurushiya, M. S. et al. Viral E3 ubiquitin ligase-mediated degradation of a cellular E3: viral $450 \quad$ mimicry of a cellular phosphorylation mark targets the RNF8 FHA domain. Mol. Cell 46, 79$45190(2012)$.

452 4. Marazzi, I. et al. Suppression of the antiviral response by an influenza histone mimic. Nature $453 \quad 483,428-433(2012)$.

454 5. Tarakhovsky, A. \& Prinjha, R. K. Drawing on disorder: How viruses use histone mimicry to 455 their advantage. J. Exp. Med. 215, 1777-1787 (2018).

456 6. Schaefer, U., Ho, J. S. Y., Prinjha, R. K. \& Tarakhovsky, A. The 'histone mimicry' by 457 pathogens. Cold Spring Harb. Symp. Quant. Biol. 78, 81-90 (2013).

458 7. Avgousti, D. C. et al. Adenovirus Core Protein VII Downregulates the DNA Damage 459 Response on the Host Genome. J. Virol. 91, (2017).

460 8. Strahl, B. D. \& Allis, C. D. The language of covalent histone modifications. Nature 403, 41-45 $461 \quad(2000)$

462 9. Jenuwein, T. \& Allis, C. D. Translating the histone code. Science 293, 1074-1080 (2001).

463 10. Berger, S. L. The complex language of chromatin regulation during transcription. Nature $464 \quad 447,407-412(2007)$.

465 11. Menachery, V. D. et al. MERS-CoV and H5N1 influenza virus antagonize antigen 466 presentation by altering the epigenetic landscape. Proc. Natl. Acad. Sci. U. S. A. 115, 467 E1012-E1021 (2018).

468 12. Schäfer, A. \& Baric, R. S. Epigenetic Landscape during Coronavirus Infection. Pathog. 469 Basel Switz. 6, (2017). 
470 13. Poppe, M. et al. The NF-kB-dependent and -independent transcriptome and chromatin 471 landscapes of human coronavirus 229E-infected cells. PLoS Pathog. 13, e1006286 (2017).

472 14. Hadjadj, J. et al. Impaired type I interferon activity and inflammatory responses in severe 473 COVID-19 patients. Science 369, 718-724 (2020).

474 15. Blanco-Melo, D. et al. Imbalanced Host Response to SARS-CoV-2 Drives Development 475 of COVID-19. Cell (2020) doi:10.1016/j.cell.2020.04.026.

476 16. Li, Y. et al. SARS-CoV-2 induces double-stranded RNA-mediated innate immune 477 responses in respiratory epithelial derived cells and cardiomyocytes. BioRxiv Prepr. Serv. $478 \quad$ Biol. (2020) doi:10.1101/2020.09.24.312553.

479 17. Chan, J. F.-W. et al. Genomic characterization of the 2019 novel human-pathogenic 480 coronavirus isolated from a patient with atypical pneumonia after visiting Wuhan. Emerg. $481 \quad$ Microbes Infect. 9, 221-236 (2020).

482 18. Yuen, K.-S., Ye, Z.-W., Fung, S.-Y., Chan, C.-P. \& Jin, D.-Y. SARS-CoV-2 and COVID483 19: The most important research questions. Cell Biosci. 10, 40 (2020).

484 19. Avgousti, D. C. et al. A core viral protein binds host nucleosomes to sequester immune 485 danger signals. Nature 535, 173-177 (2016).

486 20. Sampath, S. C. et al. Methylation of a histone mimic within the histone methyltransferase 487 G9a regulates protein complex assembly. Mol. Cell 27, 596-608 (2007).

488 21. Flower, T. G. et al. Structure of SARS-CoV-2 ORF8, a rapidly evolving coronavirus 489 protein implicated in immune evasion. BioRxiv Prepr. Serv. Biol. (2020)

$490 \quad$ doi:10.1101/2020.08.27.270637.

491 22. Muth, D. et al. Attenuation of replication by a 29 nucleotide deletion in SARS-coronavirus 492 acquired during the early stages of human-to-human transmission. Sci. Rep. 8, 15177 493 (2018).

494 23. Gordon, D. E. et al. A SARS-CoV-2 protein interaction map reveals targets for drug 495 repurposing. Nature (2020) doi:10.1038/s41586-020-2286-9. 
496 24. Zinzula, L. Lost in deletion: The enigmatic ORF8 protein of SARS-CoV-2. Biochem.

497 Biophys. Res. Commun. (2020) doi:10.1016/j.bbrc.2020.10.045.

498 25. Pereira, F. Evolutionary dynamics of the SARS-CoV-2 ORF8 accessory gene. Infect.

499 Genet. Evol. J. Mol. Epidemiol. Evol. Genet. Infect. Dis. 85, 104525 (2020).

500 26. Su, Y. C. F. et al. Discovery and Genomic Characterization of a 382-Nucleotide Deletion 501 in ORF7b and ORF8 during the Early Evolution of SARS-CoV-2. mBio 11, (2020).

502 27. Yiwen Zhang, Junsong Zhang, Yingshi Chen, Baohong Luo, Yaochang Yuan, Feng 503 Huang, Tao Yang, Fei Yu, Jun Liu, Bingfen Liu, Zheng Song, Jingliang Chen, Ting Pan, Xu

504 Zhang, Yuzhuang Li, Rong Li, Wenjing Huang, Fei Xiao, and Hui Zhang. The ORF8 Protein

505 of SARS-CoV-2 Mediates Immune Evasion through Potentially Downregulating MHC-1.

506 BioRxiv (2020).

507 28. Young, B. E. et al. Effects of a major deletion in the SARS-CoV-2 genome on the 508 severity of infection and the inflammatory response: an observational cohort study. Lancet $509 \quad$ Lond. Engl. 396, 603-611 (2020).

510 29. Li, J.-Y. et al. The ORF6, ORF8 and nucleocapsid proteins of SARS-CoV-2 inhibit type I 511 interferon signaling pathway. Virus Res. 286, 198074 (2020).

512 30. Rusinova, I. et al. Interferome v2.0: an updated database of annotated interferon513 regulated genes. Nucleic Acids Res. 41, D1040-1046 (2013).

514 31. Hou, Y. J. et al. SARS-CoV-2 Reverse Genetics Reveals a Variable Infection Gradient in 515 the Respiratory Tract. Cell 182, 429-446.e14 (2020). 


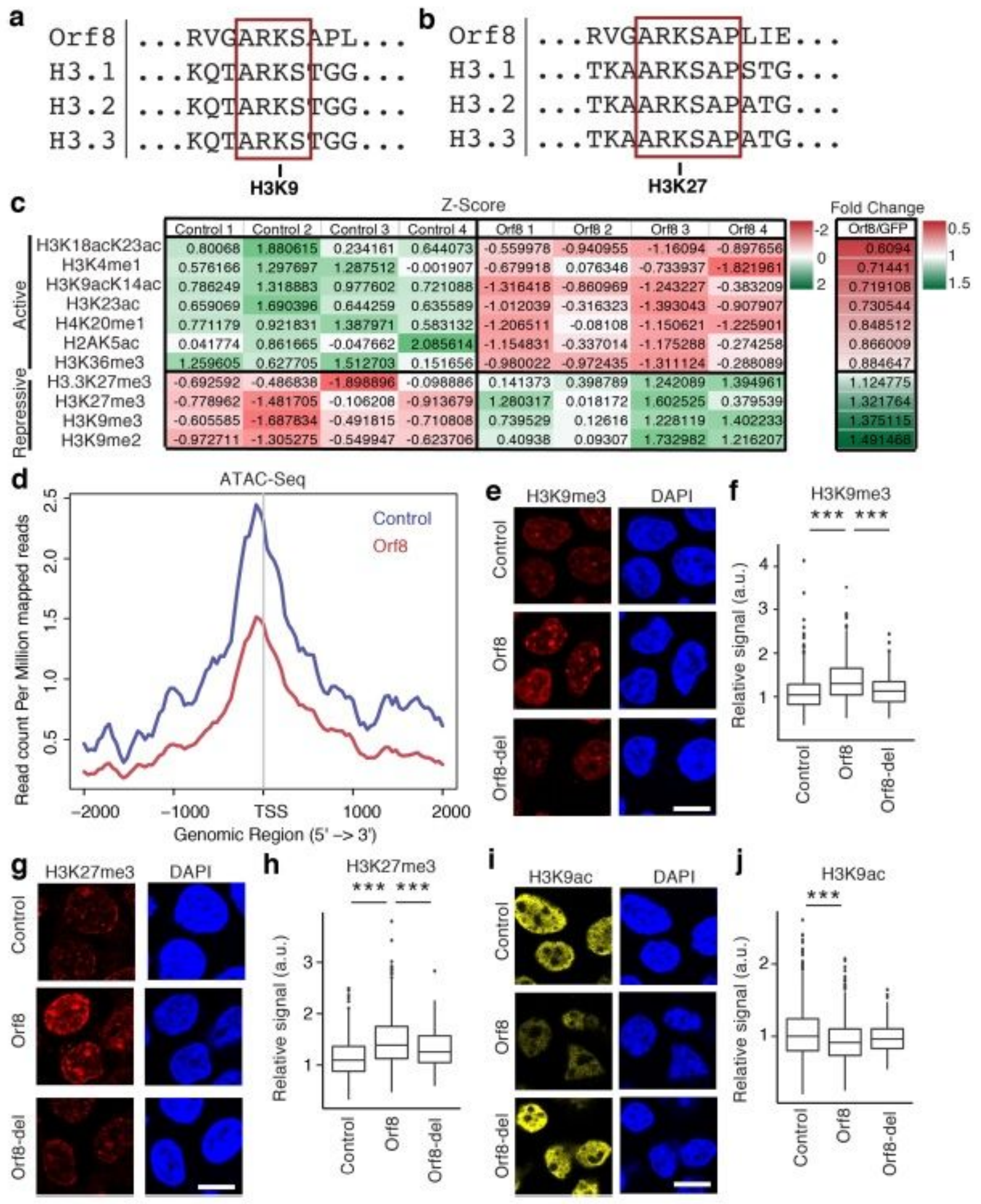

\section{Figure 1}

Orf8 histone mimicry and localization. ( $a, b)$ Orf8 contains an ARKS motif that matches the histone $\mathrm{H} 3$ tail regions surrounding the critical sites H3K9 (a) and H3K27 (b). (c) Mass spectrometry analysis of histone PTMs in control (GFP) or Orf8 expressing HEK cells isolated by FACS. Z-score and fold changes are 
shown for modifications that are significantly changed in response to Of8 expression, were detected over a minimal threshold of $1 \%$ of the total peptide abundance, and have well-established functions. Full results and raw 300 data are shown in Table S1. (d) ATAC-sequencing of 2 independent replicates of HEK cells expressing GFP or Orf8 isolated by FACS. Reads per million mapped surrounding the transcription start site (TSS) of all genes are averaged. (e-j) HEK cells transfected with GFP or Strep-Orf8 show that Orf8 expression increases H3K9me3 (e-f) and H3K27me3 ( $g-h$ ) while decreasing H3K9ac (i-j). Conversely, Orf8 with a deletion of the histone mimic site ARKSAP (Orf8-del) does not affect these histone PTMs. $\mathrm{N}=$ 614 (GFP), 497 (Orf8), 170 (Orf8-del) cells for H3K9me3; 616, 550, 154 cells for H3K27me3; 666, 568, 170 cells for H3K9ac compiled from 3 independent transfections. ***, $p<0.001,1$-way ANOVA with post-hoc 2sided t-test and Bonferroni correction. Scale bars $=5 \mu \mathrm{M}$. 


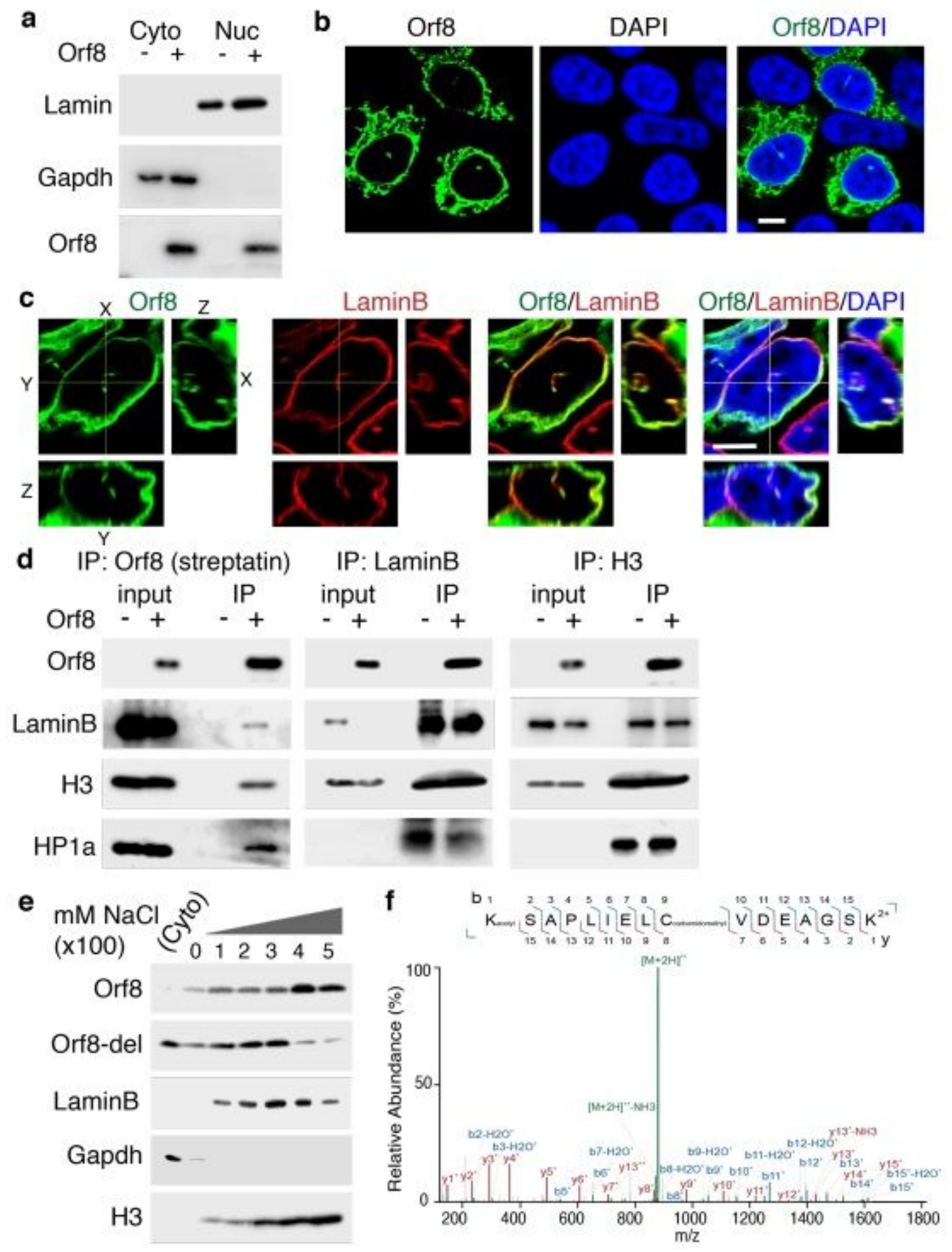

\section{Figure 2}

Mechanism of Orf8 function. (a). Subcellular fractionation of HEK cells transfected with Strep-Orf8 indicates Orf8 is present in the cytoplasm and nucleus. (b) Staining of HEK cells transfected with StrepOrf8 shows Orf8 is expressed in the cytoplasm and at the nuclear periphery as well as in nuclear puncta. (c) Orf8 colocalizes with Lamin at the nuclear periphery and within nuclear puncta. Images shown include rotation of z-stacks (right and bottom panel for each stain) to demonstrate colocalization throughout the nucleus. (d) Orf8 co-immunoprecipitates with Lamin complex-associated proteins including LaminB, HP1, 
and H3. Streptactin-conjugated beads were used for Orf8 IPs. '-' indicates cells that are not expressing Orf8 for negative control IPs performed in parallel. (e) Sequential salt extraction of HEK cells expressing Orf8 indicates Orf8 is present in chromatin fractions and dissociates from chromatin between peaks of dissociation for LaminB and histone protein H3. 'Cyto' indicates cytoplasmic fraction. (f) Targeted mass spectrometry analysis of trypsin-digested Orf8 shows Orf8 is acetylated at lysine 52 , at the site of the proposed histone mimic in Orf8. The intact $2+$ charged peptide or precursor at $879.9508++\mathrm{m} / \mathrm{z}$ was isolated and fragmented resulting in the MS/MS spectra shown. After fragmentation, the MS/MS spectra show unfragmented precursor (green) with matching product ions (b ions in blue, $y$ ions in red) within 10ppm mass error. Each fragment's intensity is given relative to the highest ion in the MS/MS spectra across the $\mathrm{m} / \mathrm{z}$ range. The color, letter, and number of each fragment indicates the sequence that fragment contains within the larger peptide (top). $Y$ fragments (red) indicate $C$ terminus matched fragments. $B$ fragments (blue) indicate $N$ terminus matched fragments. Scale bars $=5 \mu \mathrm{M}$. 

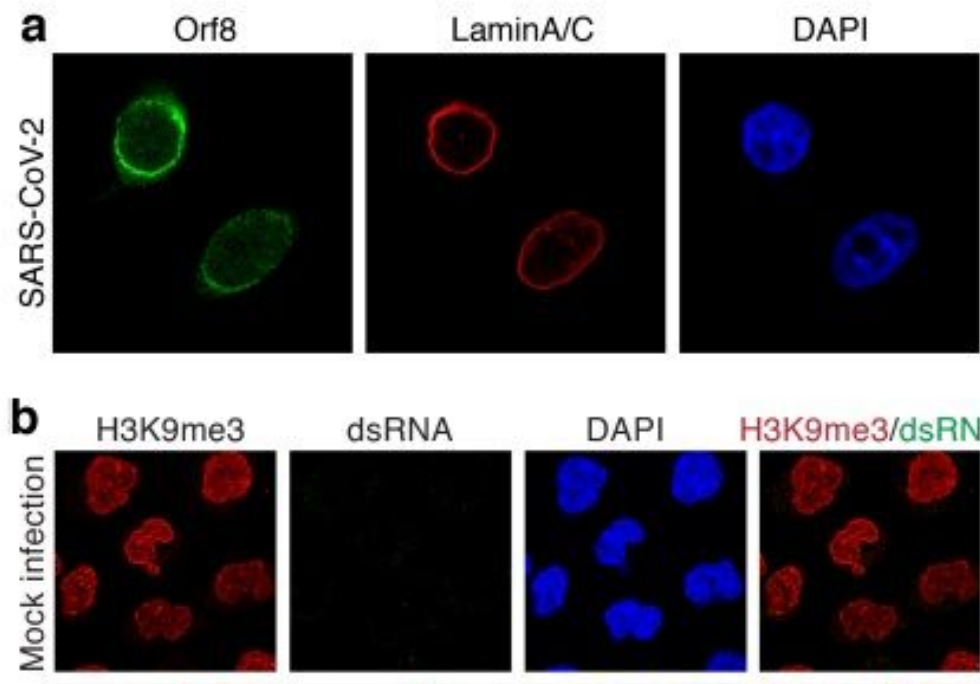

dsRNA

DAPI

H3K9me3/dsRNA C
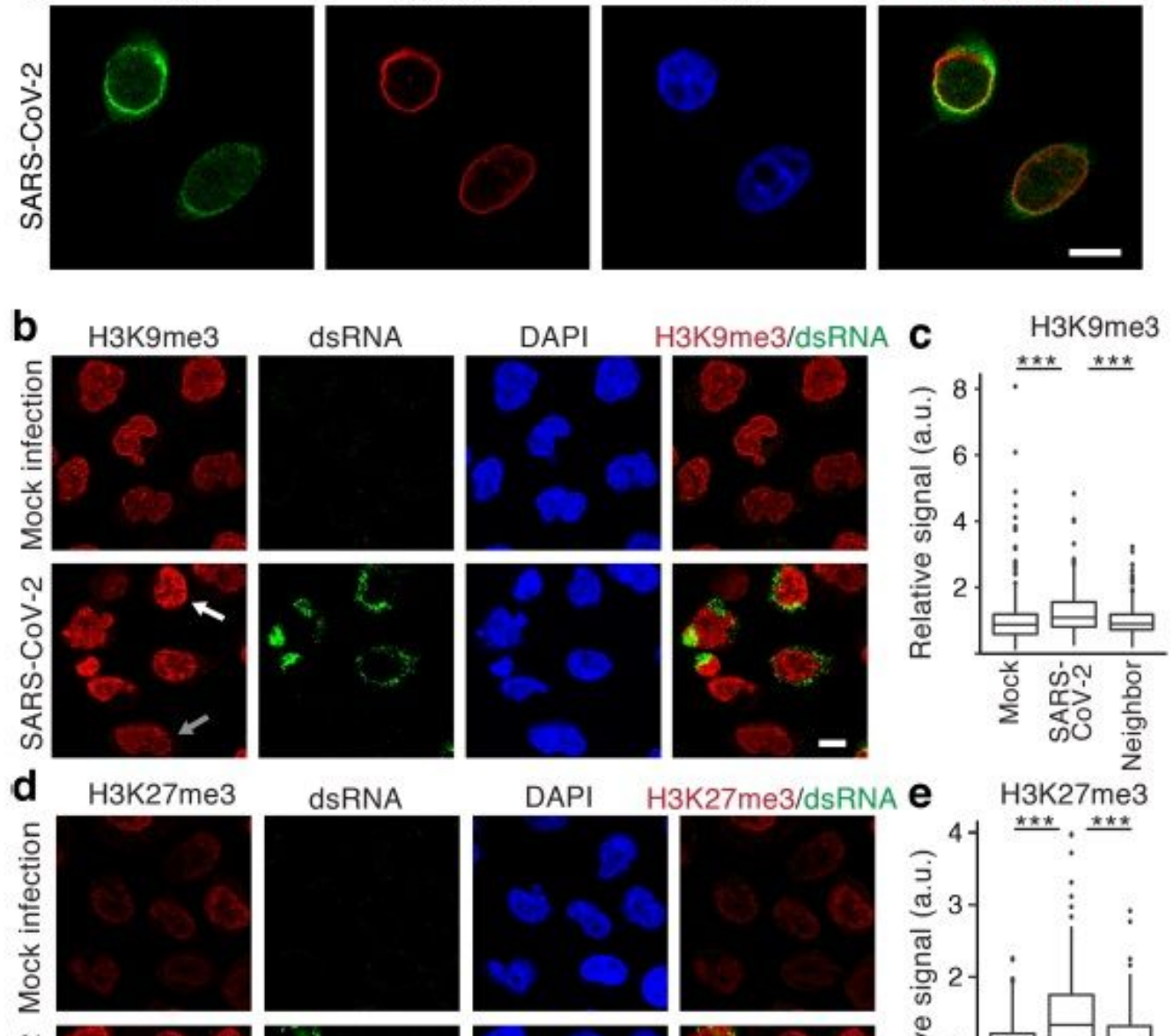

\section{DAPI}
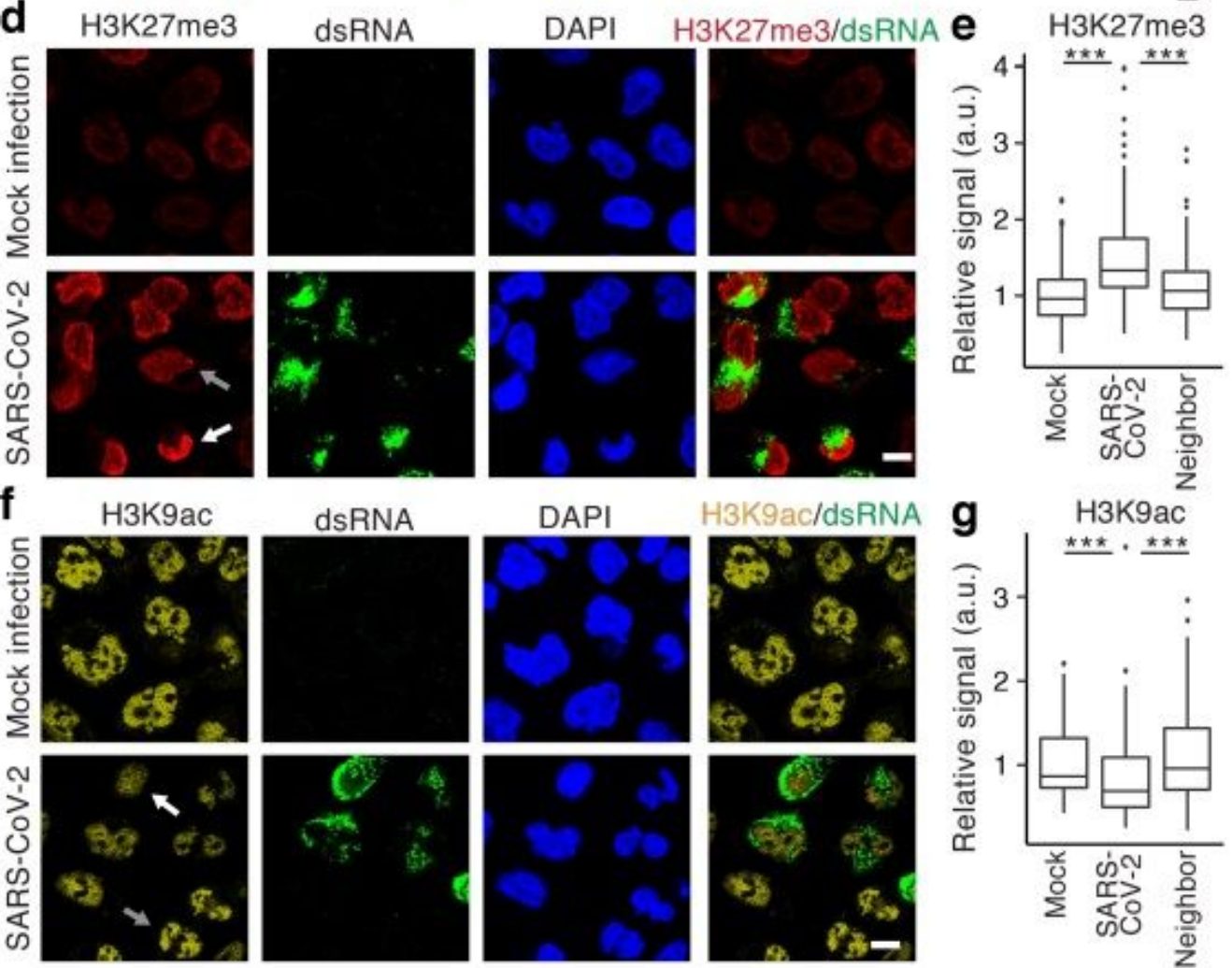

dsRNA
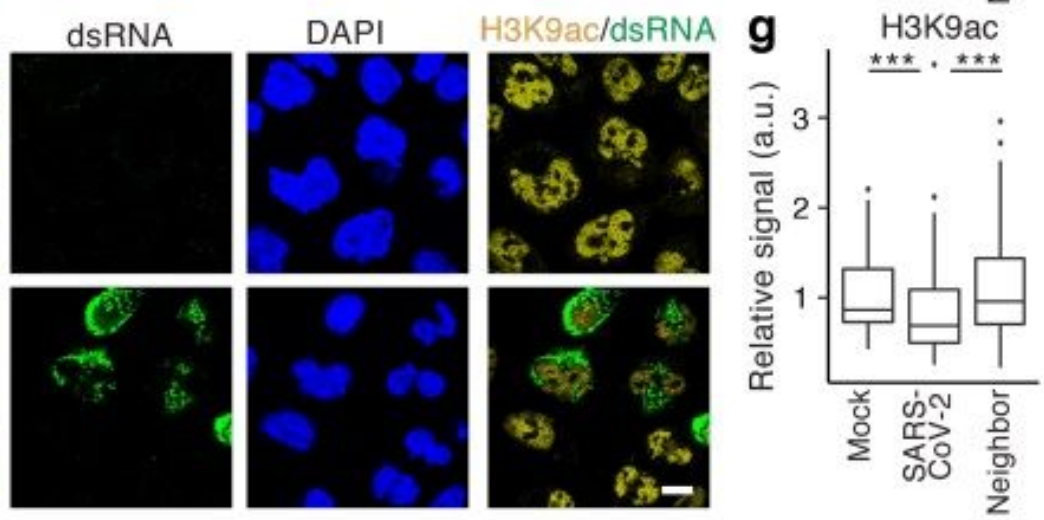

\section{Figure 3}

SARS-CoV-2 infection affects histone PTMs. (a) Orf8 and LaminA/C staining of SARS-CoV-2 infected ACEA549 cells at MOI=1, 48 hours post infection. (b) H3K9me3 staining of ACEA549 cells 48 hours after SARS-CoV-2 or mock infection at MOI=1. (338 c) Quantification of H3K9me3. N = 475 (Mock), 158 (SARSCoV-2), 265 (Uninfected neighbor) cells per condition from 3 independent infections. (d) H3K27me3 staining of ACEA549 cells 48 hours after SARS-CoV- 2 or mock infection at MOI=1. (e) Quantification of 
H3K27me3. N= 455 (Mock), 133 (SARS-CoV-2), 250 (Uninfected neighbor) cells per condition from 3 independent infections. (f) H3K9ac staining of ACEA549 cells 48 hours after SARS-CoV-2 or mock infection at MOI=1. (g) Quantification of H3K9ac. N= 385 (Mock), 177 (SARS-CoV-2), 268 (Uninfected neighbor) cells per condition from 3 independent infections. White arrows indicate infected cells. Grey arrows indicate uninfected neighbors. ${ }^{* *}, \mathrm{p}<0.001$, 1-way ANOVA with post-hoc 2-sided t-test and Bonferroni correction. Scale bars $=5 \mu \mathrm{M}$.

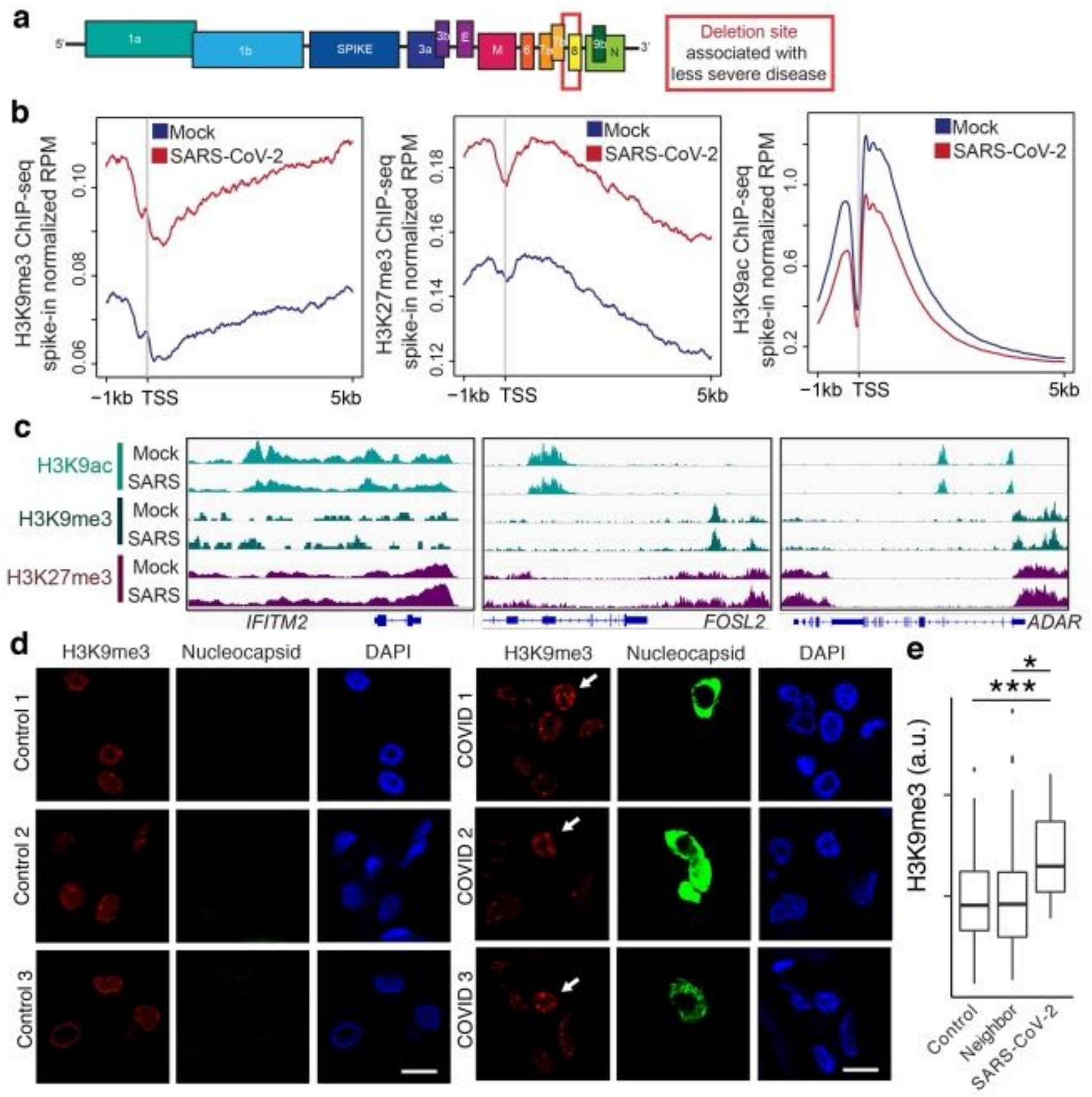

Figure 4

Genome-wide sequencing demonstrates global changes in histone PTMs in response to SARS-CoV-2 infection. (a) Model of the SARS-CoV-2 genome indicating the site of deletion that decreases disease severity in COVID-19 patients. (b) ChIP-sequencing of iPSC derived iAT2 cells fixed by 4\% PFA 48 hours 
after SARS-CoV-2 or mock infection at $\mathrm{MOI}=5$. ChIP-RX normalization shows globally increased H3K9me3 and H3K27me3 and decreased H3K9ac. $\mathrm{N}=$ at least 3 infections per ChIP fixed by 4\% PFA 48 hours after SARS-CoV-2 or mock infection at $\mathrm{MOI}=5$. (c) ChIP-sequencing gene tracks for interferon response genes with limited responses to SARS-CoV-2 infection. (d) Postmortem COVID-19 358 patient lung tissue stained for H3K9me3 and Nucleocapsid protein to identify SARS-CoV-2 infected cells. (e) Quantification of $\mathrm{H} 3 \mathrm{~K} 9 \mathrm{me} 3$ in infected cells compared to neighboring cells from the same tissue slice. $\mathrm{N}$ $=12$ SARS-CoV-2 infected cells and 131 uninfected neighboring cells from 3 COVID patient samples and 60 cells from 3 control patients. 1-way ANOVA with post-hoc 2-sided t-test and Bonferroni correction. Scale bar $=10 \mu \mathrm{M}$.

\section{Supplementary Files}

This is a list of supplementary files associated with this preprint. Click to download.

- SupplementalFigures.pdf

- TableS1HistonePTMs.xlsx

- TableS2Orf8bindingpartnerIP.xlsx

- NATUREemailattachmentSMC16092415661.pdf 